Pacific Journal of Mathematic 


\title{
SEMIGROUPS WITH DIMINISHING ORBITAL DIAMETERS
}

\author{
Mo TAK KIANG
}

In this paper, fixed point theorems for semigroups of selfmappings on a metric space $(X, d)$ subject to conditions on the size of the orbits are considered.

The concepts of diminishing orbital diameters (d.o.d.) for semigroups of mappings on a metric space and that of convex diminishing orbital diameters (c.d.o.d.) for semigroups of mappings on a convex subset of a normed linear space are introduced. Also discussed are the concepts of linearly ordered semigroups and in particular those that are Archimedean at some of its members. Certain results of Belluce and Kirk concerning a single mapping satisfying d.o.d. are generalized. Also included are results on semigroups of self-mappings on a weakly compact, convex subset of a Banach space.

1. The concept of "diminishing orbital diameters" of a single self-mapping $f$ on a metric space $(X, d)$ was first introduced by Belluce and Kirk in their paper [1]. For any point $x \in X$, let $O(x)=$ $\left\{x, f(x), f^{2}(x), \cdots\right\}$ and $\delta[O(x)]$ denote the diameters of $O(x)$. It is clear that the sequence $\left\{\delta\left[O\left(f^{n}(x)\right)\right]: n=1,2, \cdots\right\}$ is nonincreasing. Let $r(x)=\inf \left\{\delta\left[O\left(f^{n}(x)\right)\right]: n=1,2, \cdots\right\}$, then $r(x) \geqq 0$ for every $x \in X$. The mapping $f$ is said to have diminishing orbital diameters (d.o.d.) on $X$ if and only if for every $x \in X$, the condition $r(x)<\delta[0(x)]$ holds whenever $\delta[0(x)]>0$.

In this paper, we consider semigroups $\mathscr{F}$ of self-mappings with identity. Let $\mathscr{F}(x)=\{f(x): f \in \mathscr{F}\}$ and $\mathscr{F} f(x)=\{g f(x): g \in \mathscr{F}\}$. Suppose $\delta[\mathscr{F}(x)]$ denotes the diameter of $\mathscr{F}(x)$, and $r(x)=$ $\inf \{\delta[\mathscr{F} f(x)]: f \in \mathscr{F}\}$ for every $x \in X$. $\mathscr{F}$ is said to have diminishing orbital diameters (d.o.d.) on $X$, if and only if for every $x \in X$, we have $\delta[\mathscr{F}(x)]<\infty$ and the conditions $r(x)<\delta[\mathscr{F}(x)]$ holds whenever $\delta[\mathscr{F}(x)]>0$. It is clear that if $\mathscr{F}$ is generated by a single mapping $f$, then $\mathscr{F}$ has d.o.d. implies that $f$ has d.o.d. and vice versa. When $\mathscr{F}$ is a group, it clearly fails to have d.o.d.

If $\mathscr{F}$ satisfies d.o.d., then for every $x \in X, \delta[\mathscr{F}(x)]>0$ implies that there exists $g \in \mathscr{F}$ such that $\delta[\mathscr{F} g(x)]<\delta[\mathscr{F}(x)]$, i.e. there exists $p \in \mathscr{F}(x)$ such that $\delta[\mathscr{F}(p)]<\delta[\mathscr{F}(x)]$. A requirement on $\mathscr{F}$ weaker than d.o.d., when $X$ is a convex subset of a normed linear space, is motivated by the above observation and the next example.

Example. Let $X=E^{2}$ with the sup norm metric, and $f: X \rightarrow X$ 
defined by $f(a, b)=(|b|,-b)$ for every $(a, b) \in X$.

The mapping $f$ can be easily shown to be nonexpansive. Let $\mathscr{F}$ be the semigroup generated by $f$ and the identity. For $z=(1,1)$, since $\delta[\mathscr{F}(z)]>0$ and $\mathscr{F} \mid \mathscr{F}(z)$ is a family of isometries, $\mathscr{F}$ fails to have d.o.d. However, the point $p=(1,1 / 2)$ in $\overline{c o} \mathscr{F}(z)$ satisfies $\delta[\mathscr{F}(p)]=1<2=\delta[\mathscr{F}(z)]$. In fact, every point $p \in \overline{\mathrm{co}} \mathscr{F}(z)$ satisfies $\delta[\mathscr{F}(p)]<\delta[\mathscr{F}(z)]$.

Let $X$ be a convex subset of a normed linear space and $\mathscr{F}: X \rightarrow X$ a semigroup of self-mappings. $\mathscr{F}$ is said to have convex diminishing orbital diameters (c.d.o.d.) on $X$ if and only if for every $x \in X$, $\delta[\mathscr{F}(x)]<\infty$ and the condition $\delta[\mathscr{F}(x)]>0$ implies that there exists $p \in \overline{\mathrm{co}} \mathscr{F}(x)$ such that $\delta[\mathscr{F}(p)]<\delta[\mathscr{F}(x)]$.

We introduce next the concept of a linearly ordered semigroup. This is motivated by the observation of certain properties possessed by a flow (see [4]). Let $\left\{f_{s}: s \in S\right\}$ be a set of continuous self-mappings of a subset $X$ of a Banach space $B$, where $S$ (written additively) is a commutative topological semigroup with identity element $\bar{o}$ such that $f_{\bar{o}}(x)=x$ for all $x \in B$, and $f_{s}\left(f_{t}(x)\right)=f_{t}\left(f_{s}(x)\right)=f_{t+s}(x)$ for all $x \in B$ and $s, t \in S$, and satisfying the continuity condition that for each $t \in S$, $\sup \left\{\left\|f_{t}(x)-f_{s}(x)\right\|: x \in B\right\} \rightarrow 0$, as $s \rightarrow t$. Then $\left\{f_{s}: s \in S\right\}$ is called a $S$-semigroup of operators on $X$. In the case when $S=\boldsymbol{R}^{+}$, the nonnegative real numbers with the usual topology, the semigroup $\left\{f_{s}: s \in \boldsymbol{R}^{+}\right\}$is called a flow.

The following two properties are satisfied by a flow:

(1) Let $\mathscr{F}_{t}=\mathscr{F} f_{t}=\left\{f_{a}: a \in \boldsymbol{R}^{+}+t\right\}$. For $s<t$, we have $\mathscr{F}_{t} \cong \mathscr{F}_{s}$. Hence, the linear ordering of $\boldsymbol{R}^{+}$induces a linear ordering in $\mathscr{F}$ in an obvious fashion.

(2) Let $f_{s}, f_{t} \in \mathscr{F}$, with $s \leqq t$. Suppose $s \neq 0$, then there is an integer $n$ such that $n \cdot s \geqq t$. Hence, we have $\mathscr{F}\left(f_{s}\right)^{n} \subseteq \mathscr{F} f_{t}$.

In general, let $(X, d)$ be a metric space and $\mathscr{F}$ a semigroup of self-mappings on $X . \mathscr{F}$ will be called linearly ordered if it satisfies the condition that for every $f, g \in \mathscr{F}$, either $\mathscr{F} f \subseteq \mathscr{F} g$ or $\mathscr{F} g \subseteq \mathscr{F} f$. In the case when $\mathscr{F} f \subseteq \mathscr{F} g$, we say that $f$ follows $g$ and denote this fact by $f \geqq g$. Let $\mathscr{F}$ be a linearly ordered semigroup. $\mathscr{F}$ is said to be Archimedean at $g \in \mathscr{F}$ with $g \neq I d$ (the identity mapping) if for every $f \in \mathscr{F}$ with $g \leqq f$, there exists a positive integer $n$ such that $g^{n} \geqq f$. The semigroup $\mathscr{F}$ is said to be linearly ordered, Archimedean, if $\mathscr{F}$ is Archimedean at each $g \in \mathscr{F}$ where $g \neq I d$.

Clearly a flow and also any semigroup with a single generator are linearly ordered, Archimedean semigroups.

2. In this section, results of Belluce and Kirk [1], [5], concern- 
ing mappings satisfying diminishing orbital diameters (d.o.d.) are generalized.

For a single mapping $f$ on a metric space, Belluce and Kirk [1] showed that the condition that the mapping $f$ satisfies d.o.d. is sufficient for it to have a fixed point if $f$ is furthermore nonexpansive and possesses a $f$-closure point. It was shown later by Kirk [5] that for a compact metric space, this condition guarantees a fixed point when nonexpansiveness of $f$ is replaced by continuity.

We generalize the above results on a single mapping on a metric space to the case of a commutative semigroup of self-mappings having d.o.d. While in the nonexpansive case a result of Edelstein [2] is used by Belluce and Kirk to prove their result for a single mapping, the following proposition uses a result of Holmes and Narayanaswami [3] concerning commutative asymptotically-nonexpansive semigroups of self-mappings.

A semigroup $\mathscr{F}^{-}:(X, d) \rightarrow(X, d)$ is called asymptotically-nonexpansive iff for every $x, y \in X$, there exists $g \in \mathscr{F}$ such that $d[f g(x), f g(y)] \leqq$ $d(x, y)$ for all $f \in \mathscr{F}$.

The set $\{z \in X$ : there exists $x \in X$ such that for every $f \in \mathscr{F}$, $\varepsilon>0$, there exists $g \in \mathscr{F}$ with $d[f g(x), z]<\varepsilon\}$ is called the $\mathscr{F}$-closure of $X$ and is denoted by $X$.

Lemma 2.1. (Proposition 2 in [3]). Let $(X, d)$ be a commutative semigroup of continuous asymptotically-nonexpansive mappings on $X$. If $z \in X^{\Im}$, then $\mathscr{F} \mid \mathscr{F}(z)$ is a family of isometries.

Proposition 1. Let $X$ be a metric space, $\mathscr{F}$ a commutative semigroup of continuous asymptotically-nonexpansive self-mappings on $X$. If $\mathscr{F}$ has diminishing orbital diameters (d.o.d.) and there exists $z \in X^{\mathscr{\sigma}}$, then $z$ is a common fixed points of $\mathscr{F}$.

Proof. By the preceding lemma, $\mathscr{F} \mid \mathscr{F}(z)$ is a family of isometries. Hence, we have

$$
\begin{aligned}
\delta\left[\mathscr{F}^{-} f(z)\right] & =\sup \left\{d\left[g f(z), g^{\prime} f(z)\right]: g, g^{\prime} \in \mathscr{F}\right\} \\
& =\sup \left\{d\left[f g(z), f g^{\prime}(z)\right]: g, g^{\prime} \in \mathscr{F}\right\} \\
& =\sup \left\{d\left[g(z), g^{\prime}(z)\right]: g, g^{\prime} \in \mathscr{F}\right\} \\
& =\delta[\mathscr{F}(z)] .
\end{aligned}
$$

Since the above is true for all $f \in \mathscr{F}$, we have

$$
r(z)=\inf \{\delta[\mathscr{F} f(z)]: f \in \mathscr{F}\}=\delta[\mathscr{F}(z)] \cdot
$$

As $\mathscr{F}$ has d.o.d., we have $\delta[\mathscr{F}(z)]=0$, showing that $z$ is a common fixed point of $\mathscr{F}$. 
The following corollary is immediate.

CoROllary. Let $X$ and $\mathscr{F}$ be as in Proposition 1 and replace the condition that $\mathscr{F}$ has d.o.d. by the condition that $\mathscr{F}$ is nonisometric on the orbit of some point $z \in X^{\mathscr{T}}$ (i.e. there exists $g \in \mathscr{F}$ and points $x, y$ in the orbit of some point $z$ in $X^{\sigma}$ such that $d[g(x), g(y)] \neq$ $d(x, y))$. Then $z$ is a common fixed point of $\mathscr{F}$.

Theorem 2.2. Let $X$ be a compact metric space, $\mathscr{F}: X \rightarrow X a$ commutative semigroup of continuous mappings with d.o.d. Then for every $x \in X$, there exists $z \in \bigcap_{f \in \mathscr{F}} \overline{\mathscr{F} f(x)}$ such that $z$ is a common fixed point of $\mathscr{F}$.

Proof. For every $x \in X$, by the commutativity of $\mathscr{F}$ and the compactness of $X, \bigcap_{f \in \mathscr{F}} \overline{\mathscr{F} f(x)} \neq \varnothing$. Let $A=\bigcap_{f \in \mathscr{F}} \overline{\mathscr{F} f(x)}$. Then $A$ is a nonempty closed (and hence compact) subset which is invariant under $\mathscr{F}$.

By Zorn's lemma, there is a nonempty, minimal closed subset $K$ of $A$ which is invariant under $\mathscr{F}$. Suppose $\delta[K]>0$. Let $z \in K$, then $\overline{\mathscr{F}}(z)$ is a closed subset of $A$ which is invariant under $\mathscr{F}$. Hence $K=\overline{\mathscr{F}(z)}$.

Since $\mathscr{F}$ has d.o.d., we have $r(z)=\inf \{\delta[\mathscr{F} f(z)]: f \in \mathscr{F}\}<$ $\delta[\mathscr{F}(z)]=\delta[K]$. Hence, there exists $h \in \mathscr{F}$ such that $\delta[\mathscr{F} h(z)]<$ $\delta[\mathscr{F}(z)]$ which shows that $\delta[\overline{\mathscr{F} h(z)}]<\delta[\overline{\mathscr{F}(z)}]$. Consequently, $\overline{\mathscr{F} h(z)}$ is a proper closed subset of $K$ which is invariant under $\mathscr{F}$, which is impossible. This contradiction shows that $\delta[K]=\delta[\mathscr{F}(z)]=0$. Hence, $z$ is a common fixed point of $\mathscr{F}$.

3. In the case when $X$ is a weakly compact subset of a Banach space, the following is known (cf. Corollary 2 to Theorem 2 in [1]):-

Let $X$ be a nonempty, convex, weakly compact subset of a Banach space and $f$ a nonexpansive self-mapping on $X$. Suppose $f$ has d.o.d., then $f$ has a fixed point in $X$.

The assumption of convexity of $X$ was removed later by Kirk in [6] where he proved the following:-

Proposition 2 (Theorem 5 in [6]). Suppose $X$ is a nonempty, weakly compact subset of a Banach space. If $f: X \rightarrow X$ is nonexpansive and has d.o.d., then $f$ has a fixed point in $X$.

We proceed to prove a related result for semigroups of selfmappings.

Lemma 3.1. Let $(X, d)$ be a metric space and $\mathscr{F}: X \rightarrow X$ a line- 
arly ordered semigroup of mappings. Suppose $\mathscr{F}$ has d.o.d. and there exists a $g \in \mathscr{F}$ with $g \neq I d$ such that

(i) $g$ has a fixed point

(ii) $\mathscr{F}$ is Archimedean at $g$.

Then $\mathscr{F}$ has a common fixed point.

Proof. Let $z \in X$ be a fixed point of $g$. If $\mathscr{F}(z)$ is a singleton, then $z$ is a common fixed point of $\mathscr{F}$. Suppose $\delta[\mathscr{F}(z)]>0$. Since $\mathscr{F}$ has d.o.d., $\inf \{\delta[\mathscr{F} f(z)]: f \in \mathscr{F}\}<\delta[\mathscr{F}(z)]$. This implies that there exists a mapping $h \in \mathscr{F}$ such that $\delta[\mathscr{F} h(z)]<\delta[\mathscr{F}(z)]$. However, by (ii), there exists an integer $n$ such that $g^{n} \geqq h$, i.e. $\mathscr{F} g^{n} \subseteq \mathscr{F} h$. Hence, we obtain $\mathscr{F} g^{n}(z) \subseteq \mathscr{F} h(z) \subseteq \mathscr{F}(z)=\mathscr{F} g^{n}(z)$, showing that $\mathscr{F}(z)=\mathscr{F} h(z)$, which is a contradiction.

THeOREm 3. Let $X$ be a nonempty weakly compact subset of a Banach space and $\mathscr{F}: X \rightarrow X$ be a linearly ordered semigroup of mappings. Suppose $\mathscr{F}$ has d.o.d. and there exists a $g \in \mathscr{F}$ with $g \neq I d$ such that

(i) $g$ is a nonexpansive mapping with d.o.d.

(ii) $\mathscr{F}$ is Archimedean at $g$.

Then $\mathscr{F}$ has a common fixed point.

Proof. By Proposition 2, the mapping $g$ has a fixed point in $X$. By the preceding lemma, $\mathscr{F}$ has a common fixed point.

Corollary. Let $X$ be as in Theorem 3. Suppose $\mathscr{F}: X \rightarrow X$ is a flow having d.o.d. If there exists a mapping $g \in \mathscr{F}$ with $g \neq I d$ such that $g$ has a fixed point, then $\mathscr{F}$ has a common fixed point.

Proof. Since a flow is a linearly ordered, Archimedean semigroup, the result is immediate from the lemma.

3.2. The weaker hypothesis of c.d.o.d. is used in the next result. We proceed by first proving a lemma.

Lemma. Let $X$ be a nonempty, convex, weakly compact subset of a Banach space. Suppose $\mathscr{F}: X \rightarrow X$ is a commutative lineary ordered semigroup of nonexpansive mappings with $\mathscr{F}$ having c.d.o.d. If for every weakly closed $\mathscr{F}$-invariant subset $K_{0}$ of $X$ there exists a member $g_{0} \in \mathscr{F}$ with $g_{0} \neq I d$ such that

(i) $g_{0}$ has a fixed point in $K_{0}$

(ii) $\mathscr{F}$ is Archimedean at $g_{0}$, then $\mathscr{F}$ has a common fixed point. 
Proof. Let $K$ be a nonempty convex subset which is minimal with respect to being weakly closed and invariant under $\mathscr{F}$. If $\delta[K]=0$, we immediately obtain a common fixed point of $\mathscr{F}$.

Suppose $\delta[K]>0$. By the hypotheses of the lemma, there exists a mapping $g_{0} \in \mathscr{F}$ with $g_{0} \neq I d$, such that $\mathscr{F}$ is Archimedean at $g_{0}$, and a point $z \in K$ such that $g_{0}(z)=z$. Suppose $z$ is not a common fixed point of $\mathscr{F}$, then $\delta[\mathscr{F}(z)]>0$. Since $\mathscr{F}$ has c.d.o.d., there exists a point $p \in \overline{\mathrm{co}} \mathscr{F}(z) \subseteq K$ such that $\delta[\mathscr{F}(p)]<\delta[\mathscr{F}(z)]$. Hence there exists $r>0$ such that $\delta[\mathscr{F}(p)]<r<\delta[\mathscr{F}(z)]$.

Let $C_{g}(p)=\bigcap_{f \in s} \bar{B}(f g(p), r)$ and $U=\mathbf{U}_{g \in s} C_{g}(p)$. Since for each $g \in \mathscr{F}$, the set $C_{g}(p)$ contains $\mathscr{F}(p)$, we have $U \neq \varnothing$. We proceed to show that $U$ is convex and invariant under $\mathscr{F}$.

(1) Each $C_{g}(p)$ is clearly convex. Since $\mathscr{F}$ is linearly ordered, the collection $\left\{C_{g}(p): g \in \mathscr{F}\right\}$ is nested. Hence, $U$ is convex as the union of a nested family of convex subsets.

(2) Let $x \in U$, then $x \in C_{g}(p)$, for some $g \in \mathscr{F}$, i.e. $\|x-f g(p)\| \leqq r$, for all $f \in \mathscr{F}$. Since each $t \in \mathscr{F}$ is nonexpansive, we have

$$
\|t(x)-t f g(p)\| \leqq\|x-f g(p)\| \leqq r,
$$

for all $f \in \mathscr{F}$. Hence,

$$
t(x) \in \bigcap_{f \in \mathscr{F}} \bar{B}(t f g(p), r)=C_{t g}(p) \subseteq U,
$$

showing that $U$ is invariant under $\mathscr{F}$.

Consequently, (1) and (2) imply that $\bar{U}$ is closed, convex and invariant under $\mathscr{F}$.

Since $\bar{U} \cap K \supseteqq \mathscr{F}(p)$, and $K$ is invariant under $\mathscr{F} . \bar{U} \cap K$ is a nonempty, closed, convex, weakly compact subset in $K$ which is invariant under $\mathscr{F}$. By the minimality of $K$ we have $K \cap \bar{U}=K$, which implies that $K \cong \bar{U}$.

Next, we proceed to show that $\bigcap_{q \in \sim(z)} \bar{B}(q, r) \neq \varnothing$. Let $q \in \mathscr{F}(z)$, then $q \in K \subseteq \bar{U}$. For any $\varepsilon>0$, there is $q^{\prime} \in U$ such that $\left\|q-q^{\prime}\right\|<\varepsilon$. Now $q^{\prime} \in U$ implies that $q^{\prime} \in C_{h_{\varepsilon}}(p)$, for some $h_{\varepsilon} \in \mathscr{F}$. This shows that $\left\|q^{\prime}-f h_{\varepsilon}(p)\right\| \leqq r$ for all $f \in \mathscr{F}$. Hence, $\left\|q-f h_{\varepsilon}(p)\right\|<r+\varepsilon$, for all $f \in \mathscr{F}$, which implies that $\bar{B}(z, r+\varepsilon) \supseteqq \mathscr{F} h_{\varepsilon}(p)$. This shows that $\bar{B}(z, r+\varepsilon) \supseteqq \overline{\mathrm{co}} \mathscr{F} h_{\varepsilon}(p)$. Now, for each $g \in \mathscr{F}$, the set $\overline{\mathrm{co}} \mathscr{F} g(p)$ is a closed, convex subset of the weakly compact set $K$ and is therefore itself weakly compact. By the commutativity of $\mathscr{F}$, the collection $\{\overline{c o} \mathscr{F} g(p): g \in \mathscr{F}\}$ has f.i.p. Hence, $\bigcap_{g \in \mathscr{F}} \overline{\mathrm{co}} \mathscr{F} g(p) \neq \varnothing$. Consequently, we obtain $\bar{B}(q, r+\varepsilon) \supseteqq \overline{\mathrm{co}} \mathscr{F} h_{\varepsilon}(p) \supseteqq \bigcap_{g \in \mathscr{F}} \overline{\mathrm{co}} \mathscr{F} g \underline{(p)}$. Since the above result holds for every $\varepsilon>0$, we have $\bar{B}(q, r) \supseteqq \bigcap_{g \in \mathscr{F}} \mathrm{co} \mathscr{F} g(p)$. As $q$ is arbitrarily chosen from $\mathscr{F}(z)$ we obtain

$$
\bigcap_{q \in \mathscr{F}(z)} \bar{B}(q, r) \supseteqq \bigcap_{g \in \mathscr{F}} \overline{\mathrm{co}} \mathscr{F} g(p) \text {. }
$$

As $\bigcap_{g \in \mathscr{F}} \overline{\operatorname{co}} \mathscr{F} g(p) \subseteq K$, the set $A=\bigcap_{q \in \mathscr{F}(z)}[\bar{B}(q, r) \cap K]$ is nonempty. 
That $A$ is a proper, convex, weakly closed, $\mathscr{F}$-invariant subset of $K$ can be shown as follows:-

(i) Since $r<\delta[\mathscr{F}(z)], A$ is a proper subset of $K$.

(ii) Since each $\bar{B}(q, r) \cap K$ (where $q \in \mathscr{F}(z)$ ) is weakly closed, $A$ is weakly closed.

(iii) To show that $A$ is invariant under $\mathscr{F}$, it suffices to show that $\bigcap_{q \in \mathscr{T}(z)} \bar{B}(q, r)$ is invariant under $\mathscr{F}$.

Now, for any $x \in \bigcap_{q \in \mathscr{F}(z)} \bar{B}(q, r)$, we have $\|x-q\| \leqq r$ for all $q \in \mathscr{F}(z)$. Let $h \in \mathscr{F}$. For any $q \in \mathscr{F}(z)$, we have $q=f(z)$ for some $f \in \mathscr{F}$.

(a) For the case when $\mathscr{F} f \subseteq \mathscr{F}: h$

Since $f=f^{\prime} h$ for some $f^{\prime} \in \mathscr{F}$ we have

$$
\|h(x)-q\|=\|h(x)-f(z)\|=\left\|h(x)-h f^{\prime}(z)\right\| \leqq\left\|x-f^{\prime}(z)\right\| \leqq r .
$$

(b) For the case when $\mathscr{F} h \subseteq \mathscr{F} f$ :-

Since $z$ is a fixed point of $g_{0}$, we have $q=f(z)=f g_{0}^{n}(z)$ for any $n \in N$.

(1) Suppose $\mathscr{F} f g_{0} \leqq \mathscr{F} h$. As in (a), we obtain

$$
\|h(x)-q\|=\left\|h(x)-f g_{0}(z)\right\| \leqq r .
$$

(2) Suppose $\mathscr{F} f g_{0} \supseteqq \mathscr{F} h$, then $\mathscr{F} g_{0} \supseteqq \mathscr{F} h$, i.e. $g_{0} \leqq h$. Since $g_{0} \neq I d$ and $\mathscr{F}$ is Archimedean at $g_{0}$, there exists an integer $j$ such that $g_{0}^{j} \geqq h$. This shows that $\mathscr{F} g_{0}^{j} \subseteq \mathscr{F} h$, or $\mathscr{F} f g_{0}^{j} \cong \mathscr{F} h$. Hence $f g_{0}^{j}=f^{\prime \prime} h$ for some $f^{\prime \prime} \in \mathscr{F}$, which implies that

$$
\|h(x)-q\|=\left\|h(x)-f g_{0}^{j}(z)\right\|=\left\|h(x)-f^{\prime \prime} h(z)\right\| \leqq r .
$$

Hence, $\|h(x)-q\| \leqq r$ for all $q \in \mathscr{F}(z)$ in either case (a) or (b), i.e. $h(x) \in \bigcap_{q \in \mathscr{F}(z)} \bar{B}(q, r)$, showing that $A$ is invariant under $\mathscr{F}$.

However, results (i)-(iii) contradict the minimality of $K$, which shows that $K$ is necessarily a singleton, i.e. a common fixed point.

THeORem 4. Let $X$ be a nonempty, convex, weakly compact subset of a Banach space. Let $\mathscr{F}: X \rightarrow X$ be a commutative, linearly ordered semigroup of nonexpansive mappings such that $\mathscr{F}$ has c.d.o.d. Suppose $\mathscr{F}$ is Archimedean at $g_{0} \in \mathscr{F}$ with $g_{0} \neq I d$ such that $g_{0}$ has d.o.d. Then $\mathscr{F}$ has a common fixed point in $X$.

Proof. Let $K$ be a nonempty convex subset in $X$ which is minimal with respect to being weakly closed and invariant under $\mathscr{F}$. Since a mapping having d.o.d. on $X$ also has d.o.d. on every $\mathscr{F}$ invariant subset of $X, g_{0}$ has d.o.d. on $K$. By Proposition 2, $g_{0}$ has a fixed point in $K$ since $K$ is weakly compact. By the preceding lemma, $\mathscr{F}$ has a common fixed point in $X$. 
Corollary. Let $X$ be a nonempty, convex, weakly compact subset of a Banach space. Suppose $\mathscr{F}: X \rightarrow X$ is a flow of nonexpansive mappings with c.d.o.d. If there exists $g_{0} \in \mathscr{F}$ with $g_{0} \neq I d$ such that $g_{0}$ has d.o.d., then $\mathscr{F}$ has a common fixed point.

Proof. Since a flow is a commutative, linearly ordered, Archimedean semigroup, the result is immediate from Theorem 4.

4. Examples of commutative semigroups with diminishing orbital diameters can be easily constructed. The following is an example of such a semigroup.

\section{ExAmple. Let}

$$
X=\left\{\left(0, \frac{1}{2^{n}}\right): n=0,1, \cdots\right\} \cup\left\{\left(\frac{1}{2^{n}}, \frac{1}{2^{n}}\right): n=0,1, \cdots\right\} \cup(0,0) .
$$

Suppose $\left\{f_{n}: n=0,1, \cdots\right\}$ is a family of mappings defined by:

$$
\begin{aligned}
f_{n}(x, y) & =\left(0, \frac{1}{2^{n}}\right), \text { where } y>\frac{1}{2^{n}} \\
f_{n}\left(0, \frac{1}{2^{n}}\right) & =\left(\frac{1}{2^{n}}, \frac{1}{2^{n}}\right), \\
f_{n}\left(\frac{1}{2^{n}}, \frac{1}{2^{n}}\right) & =\left(0, \frac{1}{2^{n}}\right) \text { and } \\
f_{n}(x, y) & =(x, y) \text { where } y<\frac{1}{2^{n}} .
\end{aligned}
$$

Since $\left\{f_{n}: n=0,1, \cdots\right\}$ is a commutative family, the semigroup $\mathscr{F}$ generated by it and the identity mapping is commutative. Each $f_{n}$ is nonexpansive and fails to have d.o.d. while $\mathscr{F}$ clearly satisfies d.o.d. Indeed, for each point $p=(x, y)$ with $\delta[\mathscr{F}(p)]>0$, since $\mathrm{y}=1 / 2^{n}$ where $n \in\{0,1, \cdots\}$ we have $\delta\left[\mathscr{F} f_{m}(p)\right]<\delta[\mathscr{F}(p)]$ for $m>n$.

\section{REFERENCES}

1. L. P. Belluce and W. A. Kirk, Fixed point theorems for certain classes of nonexpansive mappings, Proc. Amer. Math. Soc., 20 (1969), 141-146.

2. M. Edelstein, On nonexpansive mappings, Proc. Amer. Math. Soc., 15 (1964), 684695.

3. R. D. Holmes and P. P. Narayanaswami, On asymptotically nonexpansive semigroups of mappings, Canad. Math. Bull., 13 (1970), 209-214.

4. W. A. Horn, Some fixed point theorems for compact maps and flows in Banach spaces, Trans. Amer. Math. Soc., 149 (1970), 391-404.

5. W. A. Kirk, On mappings with diminishing orbital diameters, J. London Math. Soc., 44 (1969), 107-111. 
6. - Fixed point theorems for nonexpansive mappings, A. M. S. Nonlinear Functional Analysis Vol. 18, Part I, (1970), 162-168.

Received July 12, 1971. This work was supported by a National Research Council of Canada Scholarship and NRC Grant A-3999.

The contents of this paper form part of the author's doctoral dissertation at Dalhousie University. The dissertation was prepared under the direction of Professor M. Edelstein to whom the author wishes to thank for his encouragement and advice.

DALHOUSIE UNIVERSITY 



\section{PACIFIC JOURNAL OF MATHEMATICS}

\section{EDITORS}

H. SAMELSON

Stanford University

Stanford, California 94305

C. R. Hовву

University of Washington

Seattle, Washington 98105
J. DugundJI

Department of Mathematics

University of Southern California

Los Angeles, California 90007

RICHARD ARENS

University of California

Los Angeles, California 90024

\section{ASSOCIATE EDITORS}
E. F. BECKENBACH
B. H. NeumanN
F. WOLF
K. YOSHIDA

\section{SUPPORTING INSTITUTIONS}

UNIVERSITY OF BRITISH COLUMBIA

CALIFORNIA INSTITUTE OF TECHNOLOGY

UNIVERSITY OF CALIFORNIA

MONTANA STATE UNIVERSITY

UNIVERSITY OF NEVADA

NEW MEXICO STATE UNIVERSITY

OREGON STATE UNIVERSITY

UNIVERSITY OF OREGON

OSAKA UNIVERSITY
UNIVERSITY OF SOUTHERN CALIFORNIA

STANFORD UNIVERSITY

UNIVERSITY OF TOKYO

UNIVERSITY OF UTAH

WASHINGTON STATE UNIVERSITY

UNIVERSITY OF WASHINGTON

$\stackrel{*}{*} \stackrel{*}{*} \stackrel{*}{*}{ }^{*}$ AMERICAN MATHEMATICAL SOCIETY

NAVAL WEAPONS CENTER 


\section{Pacific Journal of Mathematics}

\section{Vol. 41, No. $1 \quad$ November, 1972}

Anatole Beck and Peter Warren, Weak orthogonality.................

Jonnie Bee Bednar and Howard E. Lacey, Concerning Banach spaces whose duals are abstract $L$-spaces.............................

Louis Harvey Blake, Canonical extensions of measures and the extension of regularity of conditional probabilities..........................

R. A. Brooks, Conditional expectations associated with stochastic processes ..........................................

Theodore Allen Burton and Ronald Calvin Grimmer, On the asymptotic behavior of solutions of $x^{\prime \prime}+a(t) f(x)=e(t) \ldots \ldots \ldots \ldots \ldots \ldots$

Stephen LaVern Campbell, Operator-valued inner functions analytic on the closed disc ............................................

Yuen-Kwok Chan, A constructive study of measure theory...

Alexander Munro Davie and Bernt Karsten Oksendal, Peak interpolation sets for some algebras of analytic functions ...................

H. P. Dikshit, Absolute total-effective $\left(N, p_{n}\right)(c, 1)$ method ...............

Robert E. Edwards, Edwin Hewitt and Kenneth Allen Ross, Lacunarity for

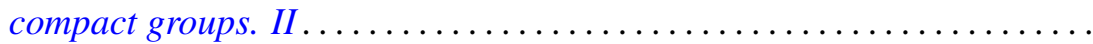

James Daniel Halpern, On a question of Tarski and a maximal theorem of Kurepa

Gerald L. Itzkowitz, A characterization of a class of uniform spaces that admit an invariant integral

Mo Tak Kiang, Semigroups with diminishing orbital diameters ..

Glenn Richard Luecke, A class of operators on Hilbert space ...

R. James Milgram, Group representations and the Adams spectral sequence. . .

G. S. Monk, On the endomorphism ring of an abelian p-group, and of a large subgroup...

Yasutoshi Nomura, Homology of a group extension ...

R. Michael Range, Approximation to bounded holomorphic functions on strictly pseudoconvex domains...

Norman R. Reilly, Inverse semigroups of partial transformations and $\theta$-classes.

Chris Rorres, Strong concentration of the spectra of self-adjoint operators

Saharon Shelah, A combinatorial problem; stability and order for models and theories in infinitary languages.

George Gustave Weill, Vector space decompositions and the abstract

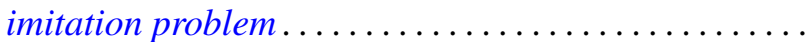

